\title{
Metabolic Syndrome and Its Components Reduce Coronary Collateralization in Chronic Total Occlusion: an Observational Study
}

Tong Liu

Beijing An Zhen Hospital: Capital Medical University Affiliated Anzhen Hospital https://orcid.org/0000-0002-9219-6084

Zheng Wu

Beijing An Zhen Hospital: Capital Medical University Affiliated Anzhen Hospital

Jinghua Liu ( $\square$ liujinghua@vip.sina.com )

Beijing An Zhen Hospital: Capital Medical University Affiliated Anzhen Hospital

Yun Lv

Beijing An Zhen Hospital: Capital Medical University Affiliated Anzhen Hospital

Wenzheng Li

Beijing An Zhen Hospital: Capital Medical University Affiliated Anzhen Hospital

\section{Original investigation}

Keywords: Chronic total occlusion, metabolic syndrome, coronary collateralization, components, score system

Posted Date: March 17th, 2021

DOI: https://doi.org/10.21203/rs.3.rs-307407/v1

License: @ (i) This work is licensed under a Creative Commons Attribution 4.0 International License. Read Full License 


\section{Abstract}

Background: Metabolic syndrome (METs) is an independent risks for the incidence of cardiovascular diseases. We investigated whether or to what extent the METs and its components was associated with coronary collateralization (CC) in chronic total occlusion (CTO).

Methods: This study involved 1709 inpatients with CTO. Data on demographic and clinical characteristics were collected by cardiovascular doctors. The CC condition was defined by Rentrop score system. Subgroup analysis, mixed models regression analysis, score systems and receiver-operating characteristic curves (ROC) analysis were done.

Results: Overall, 1709 inpatients were assigned to the Poor CC group $(n=370)$, good CC group $(n=1339)$ with or without METs. Compared to good CC, the incidence of METs was higher in poor CC for overall patients. Poor collateralization was present in $9.1 \%, 14.4 \%, 19.9 \%, 18.1 \%, 35.1 \%$ and $54.2 \%$ of the six groups, who met the diagnostic criteria of MetS $0,1,2,3,4$ and 5 times. For multivariable logistic regression, quartiles of BMI remained the risk factors of CC growth in all subgroups (adjusted $\mathrm{OR}=1.728,95 \% \mathrm{Cl} 1.518-1.967, P<0.001$ all patient group, adjusted OR $=1.827,95 \% \mathrm{Cl} 1.484-2.249, P<0.001 \mathrm{No}-\mathrm{MET}$ s group and adjusted $\mathrm{OR}=1.771,95 \% \mathrm{Cl} 1.484-2.115, P<0.001 \mathrm{MET}$ group). After adjustment for potential confounding factors, METs was an independent risk factors of CC growth in several models. Assigning a score of one for each components, this score system had significant predictive value for the CC conditions by Receiver-operating characteristic(AUC: $0.622,95 \% \mathrm{Cl}: 0.588-0.655)$.

Conclusions: METs, especially for body mass index, confers greater risk for CC formation in CTO. Score systems may help to predict CC condition.

\section{Background}

Chronic total occlusion (СТO) is defined as grade 0 thrombolysis in myocardial infarction (TIMI) flow for more than 3 months, which is frequently encountered during coronary angiography in patients with coronary artery disease (CAD) [1]. Coronary collaterals (CCs) serve as conduits that bridge occluded coronary supplied by epicardial or septal arteries, which provide an alternative source of blood supply to a myocardium subtended by an occluded vessel [2]. Well CCs can relieve angina, reduce infarct size, protect heart funtion and decrease mortality. Besides, guidewire is easy to cross and achieve recanalization effectively for good CCs [3].

Epidemiological data frequently demonstrate that metabolic syndrome (METs) is increasingly prevalent and represents an important risk factor for cardiovascular diseases, which characterized by a cluster of risk components including abdominal obesity, hyperglycemia, dyslipidemia and hypertension [4] Several groups have reported that a higher number of the metabolic syndrome components were correlated with serious CAD. Vasculogenesis, angiogenesis and arteriogenesis are three important processes for the formation of CCs, which influenced by signalling and transcriptional control, soluble mediators and their receptors, biomechanical forces and hypoxia. Abdominal obesity and dyslipidemia indicate the vascular endothelial dysfunction, which impair the processes of CCs [5]. Shen Y et al revealed that the type 2 diabetes mellitus (T2DM) adversely affected coronary collateral development through multiple cellular mechanisms [6]. There is an inverse relationship between high blood pressure (BP) and maturation of CCs [7]. Nevertheless, this result remain controversial and the the exact mechanism is still unknown.

Despite the body of evidence indicating the association between the METs and CCs, the value of the METs as a predictive biomarker for the CCs remains controversial in CTO patients, and the prognostic power of METs components is also unknown. Therefore, we investigated the association of the METs and its components with CCs development and deduced the usefulness of the METs for the diagnosis and risk assessment of CCs in clinical practice.

\section{Methods}

\section{Study population}

Overall, 1709 inpatients with chest pain who underwent coronary angiography from January 2016 to December 2019 at The Anzhen Hospital were consecutively enrolled in this study. The patients were assigned to the METs ( $n=897$ ) or No METs (control, $n=812$ ) group (Figure.1). CTO was defined as follows: 1) an occlusion lasting for more than 3 months based on the first onset of angina pectoris, previous angiogram findings, and previous infarction, and 2) TIMI grade 0 . According to the criteria of the American National Cholesterol Education Program, MetS was defined as the presence of three or more of the following criteria: body mass index (BMI) $>30 \mathrm{~kg} / \mathrm{m}^{2}$, high-density lipoprotein (HDL) $<50 \mathrm{mg} / \mathrm{dL}$ among women, and $<40 \mathrm{mg} / \mathrm{dL}$ among men, fasting plasma triglycerides (TG) $\geq 150 \mathrm{mg} / \mathrm{dL}$, systolic blood pressure (SBP) $\geq 130 \mathrm{mmHg}$, or diastolic blood pressure (DBP) $\geq 85 \mathrm{mmHg}$, fasting plasma glucose (FPG) $\geq 100 \mathrm{mg} / \mathrm{dL}$ or previously diagnosed T2DM [8]. All participants underwent bilateral coronary angiography, which was conducted by an experienced team of cardiologists. The degree of coronary collateralization was visually estimated using the Rentrop scoring system. The good collateralization was defined as graded Rentrop 2 or 3 and the poor collateralization was defined as graded Rentrop 0 or 1 [9]. The study protocol was approved by the Institutional Review Board of The Anzhen Hospital, Beijing, China.

\section{Procedure}

Data regarding the patient's sex, age, medical history, smoking status, BMI, BP, heart rate (HR), complete blood count, serum cholesterol and homocysteine concentrations were collected for all participants. The diagnostic criteria for the classic risk factors, including hypertension (HT) [10] and T2DM [11] were based on authoritative international guidelines. Blood samples was drawn from all participants and analysed by an automated biochemical analyser, which included creatinine, lipid profles, glucose and glycated albumin (GA).

\section{Statistical analyses}


Continuous variables are reported as the means \pm standard deviations for normally distributed data or medians and quartiles (quartile 1 ; quartile 3 ) for nonnormally distributed data. Discrete variables are expressed as frequencies and percentages and were compared using the chi-square test. Univariable and multivariable logistic regression analyses were performed to detect the relationship between coronary collateralization and METs. Models were adjusted for age, sex, HR, renal disease, former smoker, current smoker, prior myocardial infarction, stroke, history of percutaneous coronary intervention, history of coronary artery bypass grafting, white blood cell, hemoglobin, platelet, creatinine, eGFR, GA, uric acid, left ventricular ejection fraction (LVEF), left ventricular end-diastolic dimension, left ventricular end-systolic dimension, homocysteine, respectively. To verify the robustness of our results, subgroups analyses were performed to explore the association between the number of total MetS components and poor collateralization. These predictors of metabolic syndrome components were assigned corresponding points based on their regression coefficient and producted a score system. Receiver-operating characteristic (ROC) curves were constructed and the areas under the curves (AUCs) were calculated to assess the discriminatory power of METs for score system. A two-sided $p$ value $<0.05$ was considered statistically significant. The statistical computations were performed using SPSS software, version 23.0 (IBM Corp., Armonk, NY, USA).

\section{Results}

Among overall 1709 patients, poor CCs occurred in 230 Mets (25.6\%) and 140 non Mets patients (17.2\%), respectively $(P<0.001)$. There were no signifcant diferences in the age and gender between Mets and non Mets patients with poor CCs. Compared to good CCs, the incidence of HT and T2MD were

significantly higher in poor CCs for all patients (all $P<0.001$ ). The DBP, TG and GA levels were more elevated in patients with poor CCs for all patients (all $P<$ 0.001). Similarly, the poor CCs showed significantly higher SBP, FBG and TC levels than the Good CC for overall and METs subgroup (all $P<0.05$ ). Furthermore, there were no significant differences in medical treatments in overall group and subgroups (Table 1). 
Table 1

clinical characteristics in METs and No METs patients with poor and good collateralization.

\begin{tabular}{|c|c|c|c|c|c|c|c|c|}
\hline \multirow[t]{3}{*}{ Variables } & \multicolumn{3}{|l|}{ Overall } & \multicolumn{3}{|l|}{ METs } & \multicolumn{2}{|l|}{ No METs } \\
\hline & $\begin{array}{l}\text { Poor } \\
\text { collateralization }\end{array}$ & $\begin{array}{l}\text { Good } \\
\text { collateralization }\end{array}$ & PValue & $\begin{array}{l}\text { Poor } \\
\text { collateralization }\end{array}$ & $\begin{array}{l}\text { Good } \\
\text { collateralization }\end{array}$ & PValue & $\begin{array}{l}\text { Poor } \\
\text { collateralization }\end{array}$ & $\begin{array}{l}\text { Goc } \\
\text { coll: }\end{array}$ \\
\hline & $n=370$ & $n=1339$ & & $n=230$ & $n=667$ & & $n=140$ & $\mathrm{n}=1$ \\
\hline Male,n(\%) & $305(82.4)$ & 1107(82.7) & 0.914 & 179(77.8) & $527(79.0)$ & 0.705 & $126(90.0)$ & 580 \\
\hline Age,years & $58.0 \pm 10.5$ & $59.3 \pm 10.2$ & 0.03 & $57.5 \pm 10.6$ & $59.0 \pm 10.2$ & 0.059 & $58.8 \pm 10.4$ & 59.6 \\
\hline $\mathrm{BMI}, \mathrm{Kg} / \mathrm{m}^{2}$ & $28.2 \pm 3.7$ & $26.0 \pm 3.0$ & $<.001$ & $28.9 \pm 4.0$ & $26.9 \pm 3.1$ & $<.001$ & $27.0 \pm 2.7$ & 25.2 \\
\hline $\mathrm{HT}, \mathrm{n}(\%)$ & $265(71.6)$ & $845(63.1)$ & 0.002 & 188(81.7) & $517(77.5)$ & 0.178 & $77(55.0)$ & 328 \\
\hline T2DM,n(\%) & $162(43.8)$ & 432(32.3) & $\begin{array}{l}< \\
0.001\end{array}$ & $121(52.6)$ & 299(44.6) & 0.041 & 41(29.3) & 133 \\
\hline $\begin{array}{l}\text { Renal } \\
\text { disease,n(\%) }\end{array}$ & 11(3.0) & $27(2.0)$ & 0.269 & $8(3.5)$ & $15(2.2)$ & 0.309 & $3(2.1)$ & $1(1$. \\
\hline $\begin{array}{l}\text { Metabolic } \\
\text { syndrome,n(\%) }\end{array}$ & $230(62.2)$ & $667(49.8)$ & $<.001$ & - & - & - & - & - \\
\hline $\begin{array}{l}\text { Former } \\
\text { smoker,n(\%) }\end{array}$ & $60(16.2)$ & $217(16.2)$ & 0.996 & $34(14.8)$ & 101(15.1) & 0.895 & $26(18.6)$ & 116 \\
\hline $\begin{array}{l}\text { Current } \\
\text { smoker,n(\%) }\end{array}$ & $148(40.0)$ & $555(41.4)$ & 0.616 & $82(35.7)$ & $278(41.7)$ & 0.108 & $66(47.1)$ & 277 \\
\hline Prior MI,n(\%) & 106(28.6) & $377(28.2)$ & 0.852 & $65(28.3)$ & 201(30.1) & 0.592 & $41(29.3)$ & 176 \\
\hline Stroke,n(\%) & $20(5.4)$ & $89(6.6)$ & 0.387 & $14(6.1)$ & $49(7.3)$ & 0.519 & $6(4.3)$ & $40(t$ \\
\hline $\begin{array}{l}\text { History of } \\
\text { PCl,n(\%) }\end{array}$ & $113(30.5)$ & $405(30.2)$ & 0.913 & $74(32.2)$ & $223(33.4)$ & 0.726 & $39(27.9)$ & 182 \\
\hline $\begin{array}{l}\text { History of } \\
\text { CABG,n(\%) }\end{array}$ & $14(3.8)$ & $41(3.1)$ & 0.486 & $8(3.5)$ & $27(4.0)$ & 0.700 & $6(4.3)$ & $14(2$ \\
\hline $\mathrm{SBP}, \mathrm{mmHg}$ & $132.6 \pm 16.1$ & $127.2 \pm 16.0$ & $\dot{0} 001$ & $135.3 \pm 15.4$ & $128.5 \pm 16.3$ & $<.001$ & $128.1 \pm 16.3$ & 125 \\
\hline $\mathrm{DBP}, \mathrm{mmHg}$ & $79.5 \pm 11.7$ & $75.5 \pm 10.6$ & $<.001$ & $81.0 \pm 11.9$ & $76.1 \pm 10.6$ & $<.001$ & $77.0 \pm 11.0$ & $75 . C$ \\
\hline HR,bpm & $71.5 \pm 9.4$ & $72.0 \pm 10.5$ & 0.399 & $71.7 \pm 9.6$ & $72.6 \pm 10.5$ & 0.245 & $71.2 \pm 9.2$ & 71.4 \\
\hline $\mathrm{FBG}, \mathrm{mmol} / \mathrm{L}$ & $6.2(5.1,7.7)$ & $5.9(5.1,7.7)$ & 0.02 & $6.8(5.7,8.9)$ & $6.4(5.5,8.2)$ & 0.037 & $5.4(4.9,6.7)$ & 5.3( \\
\hline $\mathrm{TG}, \mathrm{mmol} / \mathrm{L}$ & $1.6(1.2,2.4)$ & $1.4(1.1,2.0)$ & $<.001$ & $2.0(1.6,2.8)$ & $1.9(1.3,2.5)$ & 0.002 & $1.2(1.1,1.6)$ & 1.1( \\
\hline $\mathrm{TC}, \mathrm{mmol} / \mathrm{L}$ & $4.1 \pm 1.3$ & $3.8 \pm 1.0$ & 0.001 & $4.1 \pm 1.5$ & $3.9 \pm 1.1$ & 0.006 & $4.0 \pm 1.0$ & 3.8: \\
\hline LDL-C,mmol/L & $2.3 \pm 1.0$ & $2.2 \pm 0.9$ & 0.2 & $2.3 \pm 1.1$ & $2.2 \pm 0.9$ & 0.318 & $2.4 \pm 0.9$ & 2.3: \\
\hline $\mathrm{HDL}-\mathrm{C}, \mathrm{mmol} / \mathrm{L}$ & $1.0(0.8,1.1)$ & $1.0(0.9,1.2)$ & 0.007 & $0.9(0.8,1.0)$ & $0.9(0.8,1.0)$ & 0.473 & $1.1(0.9,1.2)$ & 1.1( \\
\hline$W B C, 10^{12} / \mathrm{L}$ & $7.1 \pm 1.8$ & $7.0 \pm 1.9$ & 0.381 & $7.0 \pm 1.8$ & $7.1 \pm 1.9$ & 0.526 & $7.1 \pm 1.8$ & $6.8:$ \\
\hline $\mathrm{HGB}, \mathrm{g} / \mathrm{L}$ & 144(133,153) & $142(132,152)$ & 0.151 & 143(133,153) & $142(132,152)$ & 0.313 & $144(134,152)$ & 142 \\
\hline $\mathrm{PLT}, 10^{9} / \mathrm{L}$ & $216.7 \pm 55.0$ & $218.6 \pm 57.3$ & 0.583 & $218.8 \pm 55.7$ & $219.9 \pm 57.3$ & 0.803 & $213.4 \pm 53.7$ & 217 \\
\hline $\mathrm{Cr}, \mu \mathrm{mol} / \mathrm{L}$ & $78.5 \pm 21.3$ & $77.2 \pm 32.9$ & 0.483 & $78.5 \pm 23.8$ & $78.2 \pm 31.3$ & 0.562 & $76.9 \pm 17.3$ & $76 . \AA$ \\
\hline $\mathrm{UA}, \mu \mathrm{mol} / \mathrm{L}$ & $361.8(310.2,421.9)$ & $351.6(299.4,419.3)$ & 0.110 & $364.1(311.0,426.6)$ & $366.4(309.3,433.8)$ & 0.760 & $356.8(308.5,409.4)$ & 340 \\
\hline $\begin{array}{l}\text { eGFR, } \\
\mathrm{mL} /(\min \cdot 1.73 \\
\mathrm{m} 2)\end{array}$ & $92.8 \pm 17.5$ & $93.1 \pm 17.0$ & 0.767 & $91.7 \pm 18.9$ & $92.3 \pm 17.8$ & 0.676 & $94.6 \pm 14.9$ & $93 . \mathrm{C}$ \\
\hline $\mathrm{HCY}, \mu \mathrm{mol} / \mathrm{L}$ & $16.4 \pm 9.0$ & $16.2 \pm 9.9$ & 0.777 & $16.1 \pm 8.5$ & $15.9 \pm 9.6$ & 0.818 & $17.0 \pm 10.0$ & 16.5 \\
\hline $\mathrm{GA}, \%$ & $15.3(13.8,17.6)$ & $14.6(13.4,17.3)$ & 0.006 & $15.6(13.9,17.9)$ & $14.9(13.4,18.2)$ & 0.029 & $14.7(13.5,16.7)$ & 14.1 \\
\hline
\end{tabular}

BMI: body mass index; HT:Hypertension; T2DM: type 2 diabetes mellitus;Prior MI:Prior myocardial infarction; History of PCl: history of percutaneous coronary CABG:history of coronary artery bypass grafting; SBP: systolic blood pressure; DBP: diastolic blood pressure;HR: heart rate; FBG:Fasting blood glucose; TG: T cholesterol; LDL-C: low-density lipoprotein cholesterol; HDL-C: high-density lipoprotein cholesterol; WBC: white blood cell; HGB:hemoglobin; PLT: Platelet; Cr:cr HCY:Homocysteine; GA:Glycated albumin; LVEF:ventricular ejection fraction; LVEDd: left ventricular end-diastolic dimension; LVEDs:left ventricular end-systol inhibitors/ARBs: Angiotensin-Converting Enzyme Inhibitors/Angiotensin Receptor Blockers; CCBs:calcium channel blockers; RCA: Right coronary artery; LAD: LCX:left circumflex coronary artery 


\begin{tabular}{|c|c|c|c|c|c|c|c|c|}
\hline \multirow[t]{3}{*}{ Variables } & \multicolumn{3}{|l|}{ Overall } & \multicolumn{3}{|l|}{ METs } & \multicolumn{2}{|l|}{ No METs } \\
\hline & $\begin{array}{l}\text { Poor } \\
\text { collateralization }\end{array}$ & $\begin{array}{l}\text { Good } \\
\text { collateralization }\end{array}$ & PValue & $\begin{array}{l}\text { Poor } \\
\text { collateralization }\end{array}$ & $\begin{array}{l}\text { Good } \\
\text { collateralization }\end{array}$ & PValue & $\begin{array}{l}\text { Poor } \\
\text { collateralization }\end{array}$ & $\begin{array}{l}\text { Goc } \\
\text { coll: }\end{array}$ \\
\hline & $\mathrm{n}=\mathbf{3 7 0}$ & $n=1339$ & & $n=230$ & $n=667$ & & $n=140$ & $n=1$ \\
\hline LVEF,\% & $56.8 \pm 7.8$ & $56.2 \pm 7.8$ & 0.165 & $56.9 \pm 7.3$ & $56.6 \pm 7.7$ & 0.597 & $56.7 \pm 8.5$ & $55 . \varepsilon$ \\
\hline LVEDd,mm & $50.6 \pm 5.2$ & $51.0 \pm 5.2$ & 0.244 & $50.1 \pm 5.0$ & $50.8 \pm 5.2$ & 0.079 & $51.5 \pm 5.4$ & 51.1 \\
\hline LVEDs,mm & $35.3 \pm 5.9$ & $35.8 \pm 5.8$ & 0.159 & $35.1 \pm 5.4$ & $35.6 \pm 5.7$ & 0.278 & $35.8 \pm 6.7$ & 36.1 \\
\hline \multicolumn{9}{|l|}{$\begin{array}{l}\text { Medication,n } \\
(\%)\end{array}$} \\
\hline Antiplatelet & $370(100)$ & $1339(100)$ & 1 & $230(100)$ & $667(100)$ & 1 & $140(100)$ & 672 \\
\hline Statins & $370(100)$ & $1339(100)$ & 1 & $230(100)$ & $667(100)$ & 1 & $140(100)$ & 672 \\
\hline Diuretics & $46(12.4)$ & $167(12.5)$ & 0.984 & $34(14.8)$ & $90(13.5)$ & 0.625 & $12(8.6)$ & $77(1$ \\
\hline $\begin{array}{l}\text { ACE } \\
\text { inhibitors/ARBs }\end{array}$ & $154(41.6)$ & 494(36.9) & 0.097 & $111(48.3)$ & $290(43.5)$ & 0.208 & $43(30.7)$ & 204 \\
\hline$\beta$-blockers & $247(66.8)$ & $875(65.3)$ & 0.613 & $154(67.0)$ & $467(70.0)$ & 0.386 & $93(66.4)$ & 408 \\
\hline CCBs & $114(30.8)$ & $383(28.6)$ & 0.408 & 72(31.3) & 231(34.6) & 0.357 & $42(30.0)$ & 152 \\
\hline $\begin{array}{l}\text { hypoglycemic } \\
\text { agents }\end{array}$ & $112(30.3)$ & $334(24.9)$ & 0.039 & $86(37.4)$ & 226(33.9) & 0.335 & $26(18.6)$ & 108 \\
\hline \multicolumn{9}{|l|}{$\begin{array}{l}\text { Target vessel,n } \\
(\%)\end{array}$} \\
\hline RCA & 174(47.0) & $594(44.4)$ & 0.362 & $109(47.4)$ & $300(45.0)$ & 0.526 & $65(46.4)$ & 294 \\
\hline LAD & $136(36.8)$ & 508(37.9) & 0.678 & 91(39.6) & $238(35.5)$ & 0.273 & $45(32.1)$ & 271 \\
\hline LCX & $65(17.6)$ & $246(18.4)$ & 0.723 & $33(14.3)$ & $135(20.2)$ & 0.048 & $32(22.9)$ & 111 \\
\hline Bridege Vessel & $3(0.8)$ & $6(0.4)$ & 0.394 & $2(0.9)$ & $5(0.7)$ & 0.859 & $1(0.7)$ & $1(0$. \\
\hline $\begin{array}{l}\text { Elevated BMI,n } \\
(\%)\end{array}$ & $92(24.9)$ & 138(10.3) & $<.001$ & $87(37.8)$ & 119(17.8) & $<0.001$ & $5(3.6)$ & $19(\%$ \\
\hline $\begin{array}{l}\text { Elevated BP,n } \\
\text { (\%) }\end{array}$ & $305(82.4)$ & $991(74.0)$ & 0.001 & 216(93.9) & $584(87.6)$ & 0.007 & $89(63.6)$ & 407 \\
\hline $\begin{array}{l}\text { Elevated FBG,n } \\
(\%)\end{array}$ & $250(67.6)$ & $779(58.2)$ & 0.001 & $189(82.0)$ & $538(80.7)$ & 0.613 & 61(43.6) & 241 \\
\hline $\begin{array}{l}\text { Reduced HDL- } \\
\text { C,n (\%) }\end{array}$ & $257(69.5)$ & $863(64.5)$ & 0.073 & 202(87.8) & $863(90.4)$ & 0.266 & 55(39.3) & 260 \\
\hline $\begin{array}{l}\text { Elevated TG,n } \\
(\%)\end{array}$ & $171(46.2)$ & $466(34.8)$ & $\begin{array}{l}< \\
0.001\end{array}$ & 152(66.1) & $393(58.9)$ & 0.055 & 19(13.6) & $73(7$ \\
\hline
\end{tabular}

BMI: body mass index; HT:Hypertension; T2DM: type 2 diabetes mellitus;Prior MI:Prior myocardial infarction; History of PCl: history of percutaneous coronary CABG:history of coronary artery bypass grafting; SBP: systolic blood pressure; DBP: diastolic blood pressure;HR: heart rate; FBG:Fasting blood glucose; TG: T cholesterol; LDL-C: low-density lipoprotein cholesterol; HDL-C: high-density lipoprotein cholesterol; WBC: white blood cell; HGB:hemoglobin; PLT: Platelet; Cr:cr HCY:Homocysteine; GA:Glycated albumin; LVEF:ventricular ejection fraction; LVEDd: left ventricular end-diastolic dimension; LVEDs:left ventricular end-systol inhibitors/ARBs: Angiotensin-Converting Enzyme Inhibitors/Angiotensin Receptor Blockers; CCBs:calcium channel blockers; RCA: Right coronary artery; LAD: LCX:left circumflex coronary artery

Individuals were divided into six groups: 55 (3.2\%), 285(16.7\%), 472(27.6\%), 553 (32.4\%), 296(17.3\%) and 48 (2.8\%) who met the diagnostic criteria of MetS 0, $1,2,3,4$ and 5 times, respectively (Fig. 2). Poor collateralization was present in $9.1 \%, 14.4 \%, 19.9 \%, 18.1 \%, 35.1 \%$ and $54.2 \%$ of the six groups, which indicated the statistically differences $(P<0.05$, see Table 2$)$. The prevalence of male was the most highest in 0 group. Besides, there were significant differences of $\mathrm{GA}$ among six groups $(P<0.001)$. As the MetS components, BMI, HT, T2DM, BP, TG were increased with the increase of traits (all $P<0.001, \mathrm{Table} 2)$. 
Table 2

Baseline characteristics of the number of METs.

\begin{tabular}{|c|c|c|c|c|c|c|c|c|}
\hline \multirow[t]{3}{*}{ Variables } & \multicolumn{8}{|c|}{ the number of the presence of METs } \\
\hline & 0 & 1 & 2 & 3 & 4 & 5 & PValue & P冈0.05 \\
\hline & $\mathrm{n}=55$ & $\mathrm{n}=\mathbf{2 8 5}$ & $\mathrm{n}=472$ & $n=553$ & $n=296$ & $\mathrm{n}=48$ & & \\
\hline Male,n(\%) & $54(98.2)$ & $256(89.0)$ & 396(83.9) & $438(79.2)$ & $230(77.7)$ & $38(79.2)$ & $<0.001$ & $a, b, c, d, e, f, g, h$ \\
\hline Age, years & $59.3 \pm 9.2$ & $59.0 \pm 10.5$ & $59.8 \pm 10.3$ & $59.3 \pm 9.8$ & $57.8 \pm 11.0$ & $55.0 \pm 10.5$ & 0.013 & $\mathrm{i}, \mathrm{k}, \mathrm{l}, \mathrm{m}, \mathrm{n}$ \\
\hline $\mathrm{BMI}, \mathrm{Kg} / \mathrm{m} 2$ & $24.8 \pm 2.6$ & $25.1 \pm 2.8$ & $25.8 \pm 2.8$ & $26.5 \pm 2.9$ & $28.2 \pm 3.7$ & $32.6 \pm 2.2$ & $<0.001$ & $b, c, d, e, f, g, h . i, j, k, l, m$ \\
\hline $\mathrm{HT}, \mathrm{n}(\%)$ & $0(0)$ & $125(43.9)$ & $280(59.3)$ & 402(72.7) & $260(87.8)$ & $43(89.6)$ & $<0.001$ & $a, b, c, d, e, f, g, h, j, k, l, m$ \\
\hline T2DM,n(\%) & $0(0)$ & $31(10.9)$ & $143(30.3)$ & $236(42.7)$ & 159(53.7) & $25(52.1)$ & $<0.001$ & $a, b, c, d, e, f, g, h, j, k, l, m$ \\
\hline $\begin{array}{l}\text { Poor } \\
\text { CC,n(\%) }\end{array}$ & $5(9.1)$ & $14(14.4)$ & $94(19.9)$ & $100(18.1)$ & $104(35.1)$ & $26(54.2)$ & $<0.001$ & d,e,h,k,m,n,o \\
\hline SBP,mmHg & $117.8 \pm 8.7$ & $126.7 \pm 16.2$ & $127.0 \pm 15.9$ & $129.3 \pm 16.2$ & $132.0 \pm 16.9$ & $129.6 \pm 12.5$ & $<0.001$ & $a, b, c, d, e, g, h, j, k, l, m$ \\
\hline $\mathrm{DBP}, \mathrm{mmHg}$ & $70.8 \pm 7.2$ & $75.8 \pm 11.2$ & $75.6 \pm 10.4$ & $76.8 \pm 10.9$ & $78.1 \pm 11.7$ & $78.8 \pm 10.4$ & $<0.001$ & $a, b, c, d, e, h, k$ \\
\hline $\mathrm{HR}, \mathrm{bpm}$ & $67.8 \pm 7.1$ & $71.0 \pm 10.5$ & $72.1 \pm 10.4$ & $72.3 \pm 9.8$ & $73.0 \pm 11.3$ & $70.1 \pm 8.3$ & 0.005 & $a, b, c, d, h$ \\
\hline $\mathrm{FBG}, \mathrm{mmol} / \mathrm{L}$ & $5.1(4.7,5.4)$ & $5.2(4.8,5.6)$ & $5.6(5.1,7.5)$ & $6.3(5.3,8.3)$ & $7.1(5.9,8.8)$ & $7.5(6.5,9.7)$ & $<0.001$ & $b, c, d, e, f, g, h, i, j, k, l, m_{s}$ \\
\hline TG,mmol/L & $1.2(0.9,1.4)$ & $1.1(0.8,1.2)$ & $1.2(1.0,1.6)$ & $1.6(1.2,2.3)$ & $2.2(1.8,2.9)$ & $2.6(2.0,3.7)$ & $<0.001$ & $b, c, d, e, f, g, h, i, j, k, l, m$, \\
\hline $\mathrm{GA}, \%$ & $13.9(13.0,15.2)$ & $14.1(13.0,15.9)$ & $14.3(13.5,16.2)$ & $15.1(13.5,18.3)$ & $16.0(13.9,19.5)$ & $16.4(14.2,18.7)$ & $<0.001$ & $b, c, d, e, f, g, h, i, j, k, l, m$ \\
\hline $\mathrm{TC}, \mathrm{mmol} / \mathrm{L}$ & $3.8 \pm 0.8$ & $3.9 \pm 1.0$ & $3.8 \pm 1.1$ & $3.8 \pm 1.2$ & $4.0 \pm 1.3$ & $4.2 \pm 1.1$ & 0.021 & $\mathrm{k}, \mathrm{l}, \mathrm{m}, \mathrm{n}$ \\
\hline $\begin{array}{l}\text { LDL-C, } \\
\mathrm{mmol} / \mathrm{L}\end{array}$ & $2.2 \pm 0.8$ & $2.3 \pm 0.9$ & $2.3 \pm 1.0$ & $2.2 \pm 0.9$ & $2.3 \pm 1.0$ & $2.3 \pm 0.9$ & 0.369 & $\mathrm{~g}$ \\
\hline $\begin{array}{l}\mathrm{HDL}-\mathrm{C} \\
\mathrm{mmol} / \mathrm{L}\end{array}$ & $1.2(0.8,1.2)$ & $1.2(1.0,1.3)$ & $1.0(0.9,1.2)$ & $0.9(0.8,1.0)$ & $0.9(0.8,1.0)$ & $0.9(0.8,1.0)$ & $<0.001$ & b,c,d,e,f,g,h,i,j,k,l,m, \\
\hline LVEF,\% & $54.5 \pm 10.3$ & $56.0 \pm 8.2$ & $56.0 \pm 7.6$ & $56.7 \pm 7.4$ & $56.6 \pm 8.0$ & $56.7 \pm 7.1$ & 0.39 & \\
\hline
\end{tabular}

a:0vs1,b:0vs2,c:0vs3,d:0vs4,e:0vs5,f:1vs2,g:1vs3,h:1vs4,i:1vs5,g:2vs3,k:2vs4,l:2vs5,m:3vs4,n:3vs5,o:4vs5.

METs:Metabolic Syndrome;BMI: body mass index; HT:Hypertension; T2DM: type 2 diabetes mellitus;CC:Coronary Collateralization; SBP: systolic blood press DBP: diastolic blood pressure;HR: heart rate; FBG:Fasting blood glucose; TG: Triglycerides; TC:Total cholesterol; LDL-C: low-density lipoprotein cholesterol; HD C: high-density lipoprotein cholesterol; GA:Glycated albumin; LVEF:ventricular ejection fraction.

After adjustment for some potential risk factors, such as gender, age, smoke status, Prior myocardial infraction and LVEF, quartiles of BMI (adjusted OR = $1.728,95 \% \mathrm{Cl} 1.518-1.967, P<0.001)$, TG (adjusted OR $=1.278,95 \% \mathrm{Cl} 1.125-1.451, P<0.001)$ and SBP (adjusted OR $=1.267,95 \% \mathrm{Cl} 1.088-1.474, P=$ 0.002) remained independent determinants for poor CCs in all patients. Petients with T2MD had a signifcantly increased risk of poor CCs compared with those with no T2DM in overall, METs and no METs groups (adjusted OR = 1.894, 95\% Cl 1.387-2.586, $P<0.001$, adjusted OR $=1.908,95 \% \mathrm{Cl} 1.294-2.815, P=0.001$ and adjusted $\mathrm{OR}=2.537,95 \% \mathrm{Cl} 1.404-4.585, P=0.002)($ Table 3$)$. 
Table 3

Impact of MetS components on coronary collateral growth in patients with and without MetS.

\begin{tabular}{|c|c|c|c|c|c|c|c|c|c|c|}
\hline \multirow[t]{2}{*}{ Variables } & \multicolumn{2}{|l|}{$\begin{array}{l}\text { Quartiles of } \\
\text { components }\end{array}$} & \multicolumn{3}{|l|}{ NO MetS } & \multicolumn{3}{|l|}{ MetS } & \multicolumn{2}{|l|}{ All } \\
\hline & Range & $\mathbf{n}$ & Good/Poor & $\mathrm{OR}(95 \% \mathrm{Cl})$ & PValue & Good/Poor & OR(95\%Cl) & PValue & Good/Poor & $\mathrm{OR}(95 \% \mathrm{Cl})$ \\
\hline $\mathrm{HT}, \mathrm{n}(\%)$ & - & 1110 & $77 / 328$ & $\begin{array}{l}1.282(0.820- \\
2.004)\end{array}$ & 0.276 & $188 / 517$ & $\begin{array}{l}1.212(0.786- \\
1.870)\end{array}$ & 0.385 & $265 / 845$ & $\begin{array}{l}1.061(0.793- \\
1.419)\end{array}$ \\
\hline $\mathrm{T} 2 \mathrm{DM}, \mathrm{n}(\%)$ & - & 594 & $41 / 131$ & $\begin{array}{l}2.537(1.404- \\
4.585)\end{array}$ & 0.002 & $121 / 299$ & $\begin{array}{l}1.908(1.294- \\
2.815)\end{array}$ & 0.001 & $162 / 432$ & $\begin{array}{l}1.894(1.387- \\
2.586)\end{array}$ \\
\hline \multirow[t]{5}{*}{$\mathrm{BMI}, \mathrm{Kg} / \mathrm{m} 2$} & Per quartile & 1709 & $140 / 672$ & $\begin{array}{l}1.827(1.484- \\
2.249)\end{array}$ & $\hat{0}_{0.001}$ & $230 / 667$ & $\begin{array}{l}1.771(1.484- \\
2.115)\end{array}$ & $\hat{0}_{0.001}$ & $370 / 1339$ & $\begin{array}{l}1.728(1.518- \\
1.967)\end{array}$ \\
\hline & $\mathrm{Q} 1 \leq 24.3$ & 424 & $19 / 242$ & - & - & $21 / 142$ & - & - & $40 / 384$ & - \\
\hline & $\begin{array}{l}24.3<\mathrm{Q} 2 \leq \\
26.3\end{array}$ & 423 & $29 / 190$ & $\begin{array}{l}5.356(2.657- \\
10.801)\end{array}$ & $\begin{array}{l}< \\
0.001\end{array}$ & $30 / 174$ & $\begin{array}{l}4.918(2.690- \\
8.993)\end{array}$ & $\begin{array}{l}< \\
0.001\end{array}$ & $59 / 364$ & $\begin{array}{l}4.809(3.115- \\
7.427)\end{array}$ \\
\hline & $\begin{array}{l}26.3<Q 3 \leq \\
28.3\end{array}$ & 426 & $59 / 161$ & $\begin{array}{l}2.956(1.556- \\
5.616)\end{array}$ & 0.001 & $56 / 150$ & $\begin{array}{l}4.217(2.519- \\
7.060)\end{array}$ & $\begin{array}{l}< \\
0.001\end{array}$ & $115 / 311$ & $\begin{array}{l}3.296(2.250- \\
4.829)\end{array}$ \\
\hline & $28.3<Q 4$ & 436 & $33 / 79$ & $\begin{array}{l}1.275(0.720- \\
2.257)\end{array}$ & 0.405 & $123 / 201$ & $\begin{array}{l}1.949(1.244- \\
3.052)\end{array}$ & 0.004 & $156 / 280$ & $\begin{array}{l}1.456(1.050- \\
2.020)\end{array}$ \\
\hline \multirow[t]{5}{*}{$\mathrm{TG}, \mathrm{mmol} / \mathrm{L}$} & Per quartile & 1709 & & $\begin{array}{l}1.405(1.119- \\
1.764)\end{array}$ & 0.003 & & $\begin{array}{l}1.312(1.093- \\
1.574)\end{array}$ & 0.003 & $370 / 1339$ & $\begin{array}{l}1.278(1.125- \\
1.451)\end{array}$ \\
\hline & $\mathrm{Q} 1 \leq 1.09$ & 429 & $33 / 283$ & - & - & $21 / 92$ & - & - & $54 / 375$ & - \\
\hline & $\begin{array}{l}1.09<\mathrm{Q} 2 \leq \\
1.43\end{array}$ & 422 & $58 / 223$ & $\begin{array}{l}3.304(1.436- \\
7.063)\end{array}$ & 0.005 & $27 / 114$ & $\begin{array}{l}1.858(0.989- \\
3.489)\end{array}$ & 0.054 & $85 / 337$ & $\begin{array}{l}2.146(1.411- \\
3.264)\end{array}$ \\
\hline & $\begin{array}{l}1.43<Q 3 \leq \\
2.06\end{array}$ & 427 & $33 / 124$ & $\begin{array}{l}1.804(0.813- \\
4.000)\end{array}$ & 0.147 & 75/195 & $\begin{array}{l}2.307(1.314- \\
4.051)\end{array}$ & 0.004 & $108 / 319$ & $\begin{array}{l}1.456(1.003- \\
2.113)\end{array}$ \\
\hline & $2.06<Q 4$ & 431 & $16 / 42$ & $\begin{array}{l}1.635(0.708- \\
3.775)\end{array}$ & 0.250 & $107 / 266$ & $\begin{array}{l}1.049(0.694- \\
1.585)\end{array}$ & 0.820 & $123 / 308$ & $\begin{array}{l}1.085(0.765- \\
1.538)\end{array}$ \\
\hline \multirow[t]{5}{*}{$\begin{array}{l}\mathrm{HDL}- \\
\mathrm{C}, \mathrm{mmol} / \mathrm{L}\end{array}$} & Per quartile & 1709 & & $\begin{array}{l}0.940(0.758- \\
1.165)\end{array}$ & 0.940 & & $\begin{array}{l}0.961(0.798- \\
1.158)\end{array}$ & 0.961 & $370 / 1339$ & $\begin{array}{l}0.975(0.859- \\
1.107)\end{array}$ \\
\hline & $\mathrm{Q} 1 \leq 0.84$ & 427 & $21 / 86$ & - & - & $87 / 233$ & - & - & $108 / 319$ & - \\
\hline & $0.84<Q 2 \leq 0.98$ & 423 & $22 / 101$ & $\begin{array}{l}0.837(0.419- \\
1.671)\end{array}$ & 0.614 & $70 / 230$ & $\begin{array}{l}0.738(0.382- \\
1.426)\end{array}$ & 0.366 & $92 / 331$ & $\begin{array}{l}1.058(0.707- \\
1.584)\end{array}$ \\
\hline & $\begin{array}{l}0.98<Q 3 \leq \\
1.16\end{array}$ & 427 & $40 / 199$ & $\begin{array}{l}0.901(0.471- \\
1.725)\end{array}$ & 0.754 & $48 / 140$ & $\begin{array}{l}0.884(0.458- \\
1.705)\end{array}$ & 0.713 & $88 / 339$ & $\begin{array}{l}1.176(0.788- \\
1.758)\end{array}$ \\
\hline & $1.16<Q 4$ & 432 & $57 / 286$ & $\begin{array}{l}1.246(0.731- \\
2.124)\end{array}$ & 0.420 & $25 / 64$ & $\begin{array}{l}0.692(0.349- \\
1.372)\end{array}$ & 0.292 & $82 / 350$ & $\begin{array}{l}1.011(0.682- \\
1.499)\end{array}$ \\
\hline \multirow[t]{5}{*}{ SBP,mmHg } & Per quartile & 1709 & & $\begin{array}{l}1.091(0.855- \\
1.383)\end{array}$ & 0.482 & & $\begin{array}{l}1.417(1.157- \\
1.735)\end{array}$ & 0.001 & $370 / 1339$ & $\begin{array}{l}1.267(1.088- \\
1.474)\end{array}$ \\
\hline & $\mathrm{Q} 1 \leq 119$ & 427 & $31 / 197$ & - & - & $31 / 168$ & - & - & $62 / 365$ & - \\
\hline & $119<Q 2 \leq 128$ & 427 & $42 / 184$ & $\begin{array}{l}1.515(0.687- \\
3.341)\end{array}$ & 0.303 & $39 / 162$ & $\begin{array}{l}2.827(1.459- \\
5.475)\end{array}$ & 0.002 & $81 / 346$ & $\begin{array}{l}2.131(1.312- \\
3.462)\end{array}$ \\
\hline & $128<Q 3 \leq 138$ & 427 & $33 / 161$ & $\begin{array}{l}1.129(0.573- \\
2.226)\end{array}$ & 0.726 & $64 / 169$ & $\begin{array}{l}2.033(1.170- \\
3.534)\end{array}$ & 0.012 & $97 / 330$ & $\begin{array}{l}1.563(1.037- \\
2.357)\end{array}$ \\
\hline & $138<Q 4$ & 428 & $34 / 130$ & $\begin{array}{l}1.316(0.676- \\
2.563)\end{array}$ & 0.418 & $96 / 168$ & $\begin{array}{l}1.456(0.907- \\
2.339)\end{array}$ & 0.120 & $130 / 298$ & $\begin{array}{l}1.436(0.986- \\
2.091)\end{array}$ \\
\hline \multirow[t]{5}{*}{ DBP,mmHg } & Per quartile & 1709 & & $\begin{array}{l}1.064(0.831- \\
1.362)\end{array}$ & 0.622 & & $\begin{array}{l}1.204(0.988- \\
1.466)\end{array}$ & 0.066 & $370 / 1339$ & $\begin{array}{l}1.124(0.967- \\
1.307)\end{array}$ \\
\hline & $\mathrm{Q} 1 \leq 70$ & 403 & $29 / 173$ & - & - & $38 / 163$ & - & - & $67 / 336$ & - \\
\hline & $70 \otimes Q 2 \leq 75$ & 435 & $38 / 195$ & $\begin{array}{l}1.213(0.540- \\
2.722)\end{array}$ & 0.640 & $38 / 164$ & $\begin{array}{l}1.618(0.872- \\
3.004)\end{array}$ & 0.127 & $76 / 359$ & $\begin{array}{l}1.381(0.860- \\
2.217)\end{array}$ \\
\hline & $75<Q 3 \leq 83$ & 461 & $39 / 188$ & $\begin{array}{l}1.465(0.725- \\
2.960)\end{array}$ & 0.288 & $54 / 180$ & $\begin{array}{l}2.021(1.168- \\
3.499)\end{array}$ & 0.012 & $93 / 368$ & $\begin{array}{l}1.650(1.091- \\
2.495)\end{array}$ \\
\hline & $83<Q 4$ & 410 & $34 / 116$ & $\begin{array}{l}1.383(0.705- \\
2.711)\end{array}$ & 0.346 & $100 / 160$ & $\begin{array}{l}2.000(1.221- \\
3.276)\end{array}$ & 0.006 & $134 / 276$ & $\begin{array}{l}1.629(1.109- \\
2.394)\end{array}$ \\
\hline
\end{tabular}

OR:odds ratio; Cl:confdence interval; METs:Metabolic Syndrome; BMI: body mass index; HT:Hypertension; T2DM: type 2 diabetes mellitus; HDL-C:high-density lipoprotein cholesterol, TG:Triglycerides, FBG:Fasting blood glucose; SBP: systolic blood pressure; DBP: diastolic blood pressure.

a Multiple adjustment for gender, age, smoke status, Prior myocardial infraction, left ventricular ejection fraction. 


\begin{tabular}{|c|c|c|c|c|c|c|c|c|c|c|}
\hline \multirow[t]{2}{*}{ Variables } & \multicolumn{2}{|l|}{$\begin{array}{l}\text { Quartiles of } \\
\text { components }\end{array}$} & \multicolumn{3}{|l|}{ NO MetS } & \multicolumn{3}{|l|}{ MetS } & \multicolumn{2}{|l|}{ All } \\
\hline & Range & $\mathrm{n}$ & Good/Poor & OR(95\%Cl) & PValue & Good/Poor & OR(95\% Cl) & PValue & Good/Poor & $\mathrm{OR}(95 \% \mathrm{Cl})$ \\
\hline \multirow[t]{5}{*}{$\mathrm{FBG}, \mathrm{mmol} / \mathrm{L}$} & Per quartile & 1709 & & $\begin{array}{l}0.916(0.725- \\
1.158)\end{array}$ & 0.463 & & $\begin{array}{l}1.028(0.857- \\
1.233)\end{array}$ & 0.766 & $370 / 1339$ & $\begin{array}{l}1.028(0.898- \\
1.178)\end{array}$ \\
\hline & $\mathrm{Q} 1 \leq 5.13$ & 427 & $50 / 223$ & - & - & $37 / 107$ & - & - & $87 / 340$ & - \\
\hline & $5.13 \otimes \mathrm{Q} 2 \leq 5.95$ & 427 & $39 / 225$ & $\begin{array}{l}0.718(0.348- \\
1.484)\end{array}$ & 0.371 & $31 / 132$ & $\begin{array}{l}1.146(0.635- \\
2.066)\end{array}$ & 0.651 & $70 / 357$ & $\begin{array}{l}0.890(0.587- \\
1.349)\end{array}$ \\
\hline & $\begin{array}{l}5.95<Q 3 \leq \\
7.87\end{array}$ & 430 & $23 / 100$ & $\begin{array}{l}1.031(0.490- \\
2.171)\end{array}$ & 0.936 & $81 / 226$ & $\begin{array}{l}1.141(0.651- \\
2.000)\end{array}$ & 0.644 & $104 / 326$ & $\begin{array}{l}1.168(0.768- \\
1.775)\end{array}$ \\
\hline & $7.87<Q 4$ & 425 & $28 / 114$ & $\begin{array}{l}0.840(0.409- \\
1.722)\end{array}$ & 0.634 & $81 / 202$ & $\begin{array}{l}0.966(0.625- \\
1.492)\end{array}$ & 0.877 & $109 / 316$ & $\begin{array}{l}0.976(0.679- \\
1.402)\end{array}$ \\
\hline
\end{tabular}

OR:odds ratio;Cl:confdence interval; METs:Metabolic Syndrome; BMI: body mass index; HT:Hypertension; T2DM: type 2 diabetes mellitus; HDL-C:high-density lipoprotein cholesterol, TG:Triglycerides, FBG:Fasting blood glucose; SBP: systolic blood pressure; DBP: diastolic blood pressure.

a Multiple adjustment for gender, age, smoke status, Prior myocardial infraction, left ventricular ejection fraction.

Table 4 show results of the multivariate logistic regression analysis for the prediction of poor CCs according to MetS. The ORs of incident poor CCs were 1.631 (95\% Cl 1.286-2.070), 1.639(95\% Cl 1.291-2.080), 1.642 (95\% Cl 1.292-2.087), 1.651 (95\% Cl 1.269-2.146), 1.643 (95\% Cl 1.211-2.229) for MetS in model $1,2,3,4,5$. After adjusted for elevated BMI, elevated BP, elevated FPG, reduced HDL-c, elevated TG (Model 6), the MetS was also the independent risk factors of poor CCs, which OR was 1.669 (95\% Cl 1.260-2.291).

Table 4

Odds ratio and $95 \%$ confdence interval for coronary collateral growth

\begin{tabular}{|c|c|c|}
\hline \multirow[t]{2}{*}{ Models } & \multicolumn{2}{|l|}{ METs } \\
\hline & $\mathrm{OR}(95 \% \mathrm{Cl})$ & PValue \\
\hline Model 1 & $1.631(1.286-2.070)$ & $<0.001$ \\
\hline Model 2 & $1.639(1.291-2.080)$ & $<0.001$ \\
\hline Model 3 & $1.642(1.292-2.087)$ & $<0.001$ \\
\hline Model 4 & $1.651(1.269-2.146)$ & $<0.001$ \\
\hline Model 5 & $1.643(1.211-2.229)$ & 0.001 \\
\hline Model 6 & $1.669(1.260-2.291)$ & 0.001 \\
\hline \multicolumn{3}{|c|}{ Model 1:adjusted for age, sex; } \\
\hline \multicolumn{3}{|c|}{ Model 2:adjusted for age, sex, heart rate, renal disease, former smoker, current smoker; } \\
\hline \multicolumn{3}{|c|}{$\begin{array}{l}\text { Model 3:adjusted for age, sex, heart rate, renal disease, former smoker,current smoker, prior myocardial infarction, stroke, history of percutaneous coronary } \\
\text { intervention, history of coronary artery bypass grafting; }\end{array}$} \\
\hline \multicolumn{3}{|c|}{$\begin{array}{l}\text { Model 4:adjusted for age, sex, heart rate, renal disease, former smoker,current smoker, prior myocardial infarction, stroke, history of percutaneous coronary } \\
\text { intervention, history of coronary artery bypass grafting, white blood cell, homocysteine, platelet, creatinine, eGFR, glycated albumin, uric acid; }\end{array}$} \\
\hline \multicolumn{3}{|c|}{$\begin{array}{l}\text { Model 5:adjusted for age, sex, heart rate, renal disease, former smoker,current smoker, prior myocardial infarction, stroke, history of percutaneous coronary } \\
\text { intervention, history of coronary artery bypass grafting, white blood cell, homocysteine, platelet, creatinine, eGFR, glycated albumin, uric acid,ventricular } \\
\text { ejection fraction; left ventricular end-diastolic dimension; left ventricular end-systolic dimension, homocysteine; }\end{array}$} \\
\hline
\end{tabular}

Based on the Regression Coefficeint, point was assigned to METs components and subsequently summed to obtain a total difficulty score.Elevated BMI was for 19, Elevated BP was for 8, Elevated FBG was for 7, Reduced HDL-C was for 1 and Elevated TG was for 8 (Table 5). The overall, METs and no METs groups were performed on the ROC curve (AUC: $0.622,95 \% \mathrm{Cl}$ : $0.588-0.655$, AUC: $0.651,95 \% \mathrm{Cl}$ : $0.608-0.693$ and AUC: $0.569,95 \% \mathrm{Cl}$ : 0.517-0.620) (Table 6, Fig. 3). 
Table 5

Multivariable analysis of the METs components.

\begin{tabular}{|lllll|}
\hline Variables & OR $(95 \% \mathrm{Cl})$ & $P$ Value & Regression Coefficeint & point \\
\hline Elevated BMI & $2.619(1.924-3.530)$ & $<0.001$ & 0.963 & 19 \\
\hline Elevated BP & $1.528(1.132-2.062)$ & 0.006 & 0.424 & 8 \\
\hline Elevated FBG & $1.427(1.111-1.832)$ & 0.005 & 0.355 & 1 \\
\hline Reduced HDL-C & $1.051(0.808-1.366)$ & 0.71 & 0.05 & 8 \\
\hline Elevated TG & $1.480(1.159-1.891)$ & 0.002 & 0.392 & 7 \\
\hline BMl: body mass index; BP: blood pressure; FBG:Fasting blood glucose; HDL-C: high-density lipoprotein cholesterol; TG: Triglycerides. \\
\hline
\end{tabular}

Table 6

The ROC Curve analysis of the METs with poor collateralization

\begin{tabular}{|lllllll|}
\hline Factors & AUC & $\boldsymbol{P}$ & $\mathbf{9 5 \%} \mathrm{Cl}$ & $\mathrm{Se}(\%)$ & $\mathrm{Sp}(\%)$ & Cut off point \\
\hline All Patients & 0.622 & $<0.001$ & $0.588-0.655$ & $43.0 \%$ & $76.3 \%$ & 22 \\
METs & 0.651 & $<0.001$ & $0.608-0.693$ & $35.7 \%$ & $86.2 \%$ & 31 \\
\hline No METs & 0.569 & 0.011 & $0.517-0.620$ & $59.3 \%$ & $54.2 \%$ & 9 \\
\hline
\end{tabular}

\section{Discussion}

In this cohort study, we demonstrated significantly METs values in the coronary collateral grow based on multivariable and subgroup analyses. After adjusted for confounding factors, METs was also the independent factors of poor coronary collateralization. Besides, for METs components analysis and model establish, BMI was the strongest predictor of METs components.

\section{The mechanisms of METs reduce coronary collateralization}

The underlying molecular mechanisms of reduced CCs in METs are poorly understood and regulated by multiple factors. when a coronary artery was occluded completely, intra-arterial pressure gradients and shear stress on the arterial wall were increased, which triggered inflammation, the redox state, gene expression as well as coronary collateral growth [12]. First, when an artery was occlusion, vascular channels was stimulated by shear stress-driven redirection of flow. Then, neutrophils and lymphocytes active a series of downstream molecular pathways, which increased expression of proteins involved in monocyte attraction and adherence. Next, inflammatory cytokines adhere to the endothelium and differentiate into macrophages, which paly an important role in vessel growth and development. Finally, collateral vessels increase diameter because of muscle cells proliferate and switch to a contractile phenotype [5].

The genger characteristics of METs are oxidative stress and endothelial dysfunction that effect vascular cell phenotype switch directly [13]. The ROS was over production in METs patients, which is the major cause of mitochondrial dysfunction and reduce the production of ATP. The reduction of ATP delay the phenotype switch from quiescent to proliferating and migrating phenotypes [14]. As the persistent inflammatory state in METs, the expression of proinflammatory cytokines are increase that trigger ROS production and reduce NO production, which play an vital role in oxidative stress and endothelial dysfunction [15]. Besides, those cytokines act as mediators to regulate signaling pathways of coronary collateralization growth, such as MAPK pathways and Rho/Rho kinase pathway [16].

\section{Relation between METs components and coronary collateralization}

The METs consists of five factors, insulin resistance, abdominal obesity, HT and high TG and low HDL-C levels, which indicate four central features: insulin resistance, visceral obesity, atherogenic dyslipidemia and endothelial dysfunction [17]. Those are interrelated as well as independent. In obese patients, baroreceptor sensitivity is impaire and activation of the sympathetic nervous system, which cause the incidence of HT. Besides, obesity is a risk factor of T2DM and dyslipidemia [18]. 104 obese patients divided into two groups, poor and good collateralization. BMI was higher in poor collateralization, which was negatively correlated with Rentrop score [19]. In our study, BMI was an independent risk in three study cohorts and the regression coefficeint was maximum. The gain of oxidative stress, aggravation infammatory process and reduction of pro-angiogenic factors are the major causes of poor collateralization in T2DM patients [6]. The relationship between HT and CC remains controversial. Some researchers found that high BP was positively related to a well-developed CC, which might increased perfusion and fluid shear stress [20]. While, Börekçi A et al found that there was negative relationship between $\mathrm{CC}$ and $\mathrm{HT}$. We found that SBP was an independent risk factor in all individuals [21]. A recent studies have proven that T2MD in particular affected early stages of collateral growth whereas HT has its impact on later remodeling stages [22]. Dyslipidemia play an important role in the process of endothelial cell dysfunction and arterial stifness,which associated with coronary collateral vessel growth. For HDL-C, it was positively associated with angiographic collateral score in Kadi study [23]. But, Shen $\mathrm{Y}$ et al found it was not association between serum HDL-C level and coronary collateral score [24]. In the present study, there was no significant between HDL-C level and CC. According to the regression coefficeint of components, the prognosis values were different. The sensitivity and specificity were increased by score systems.

\section{Basic and Clinical Research}


This study demonstrates that METS is associated with poor CCs robustly by Logistic analysis and ROC curve. After score model establish, we also can assess risk of poor CCs by METs components. In rate models, CC growth was maximal at 9 days of coronary occlusion in health rates but did not occur in METs rates. After given anti-miR-21 to blocked cell proliferation in METs rates, the CC growth was improved significantly. It was demonstrated that METs influenced the CC growth by blocking vascular switch [25]. Hattan $\mathrm{N}$ et al found that the reduction of oxidative stress could improve CC growth. Compared to the lean littermate

rates, the ratio of collateral-dependent zones (CZ)/ normal zone (NZ) flow was lower in METs rates. After given ecSOD and VEGF to reduce oxidative stress, the $\mathrm{CZ/NZ}$ ratio was increased [26]. A research found that MMP12 can act as a novel positive regulator that inhibited coronary collateral development in a rat model of METs. Compared to the health rate, coronary collateral was reduced in the METs rat after left artery disease occlusion. After inhibition of MMP12 to block endostatin and angiostatin, the coronary collateral was grew [27].

In clinical practice, Yilmaz MB et al enrolled 596 consecutive patients with total occlusion of the right coronary artery. Presence of MTs was significantly higher in patients with poor CCs than in those with good CCs. After regression analysis, METs was independent predictors of angiographically determined poor CCs [28]. In an observational study, the AIP was independently associated with the less developed collateral vessels, which enrolled 387 patients with at least one coronary occlusion. In multivariable analysis adjusting for potential confounding factors, METs was also an independent risks[29]. The results was further confirmed in our study. Compared those clinical studies, this study explore the weight of METs components by subgroup analysis. Besides, according to the ROC curve, the score models were targeted to different populations, included METs patients and no METs patients, which improves prediction efficacy and accuracy in clinical practice.

\section{Limitations}

Our study has some limitations. First, the single-centre nature of this study and the relatively small number of enrolled patients may have introduced selection bias. Second, the underlying mechanistic link between MetS and CC is not clear that potential risks may affect CC growth and METs state. Even though giving subgroup analysis and multivariate logistic regression analysis, It is uncertain whether we will be able to use the results for any recommendations.

Furthermore, we use Rentrop scoring system to identify the condition of CC, which is assess easily. Coronary collaterals may be more accurately assessed by hemodynamic indices.

\section{Conclusions}

In patients with chronic total occlusion, poor coronary collateralization seems tightly linked to METs, especially fin BMI, T2DM. After adjusted potential risks and established score system, METs was an independent risk factor for CC growth.

\section{Declarations}

\section{Ethics approval and consent to participate}

The study protocol was approved by the Institutional Review Board of The Anzhen Hospital, Beijing, China.

\section{Consent for publication}

Yes

\section{Availability of data and materials}

Data available on request from the authors

\section{Competing interests}

The authors declare that they have no competing interests

\section{Funding}

This study was funded by the National Natural Science Foundation of China (81970291)

\section{Authors' contributions}

Tong Liu: designed the work and wrote manuscript;

Zheng Wu: acquisition and analysis;

Jinghua Liu: substantively revised manuscript;

Yun Lv: obtain data;

Wenzheng Li: analysis data.

\section{Acknowledgements}

None 


\section{References}

1. Zhu Y, Meng S, Chen M, Liu K, Jia R, Li H, Zhu H, Jin Z. Long-term prognosis of chronic total occlusion treated by successful percutaneous coronary intervention in patients with or without diabetes mellitus: a systematic review and meta-analysis. Cardiovasc Diabetol. 2021; 20: 9

2. Aghajanian A, Zhang H, Buckley BK, Wittchen ES, Ma WY, Faber JE. Decreased inspired oxygen stimulates de novo formation of coronary collaterals in adult heart. J Mol Cell Cardiol. 2021; 150:1-11.

3. Wu K, Huang Z, Zhong Z, Liao H, Zhou Y, Luo B, Zhang B. Predictors, treatment, and long-term outcomes of coronary perforation during retrograde percutaneous coronary intervention via epicardial collaterals for recanalization of chronic coronary total occlusion. Catheter Cardiovasc Interv. 2019; 93 : 800-809.

4. Xu JJ, Song Y, Jiang P, Jiang L, Zhao XY, Gao Z, Li JX, Qiao SB, Gao RL, Yang YJ,et al. Eff ects of metabolic syndrome on onset age and long-term outcomes in patients with acute coronary syndrome. World J Emerg Med. 2021; 12: 36-41.

5. Allahwala UK, Khachigian LM, Nour D, Ridiandres A, Billah M, Ward M, Weaver J, Bhindi R.Recruitment and maturation of the coronary collateral circulation: Current understanding and perspectives in arteriogenesis. Microvasc Res.2020; 132: 104058.

6. Shen Y, Ding FH, Dai Y, Wang XQ, Zhang RY, Lu L, Shen WF. Reduced coronary collateralization in type 2 diabetic patients with chronic total occlusion. Cardiovasc Diabetol. 2018; 17: 26.

7. Shen Y, Ding FH, Wu F, Lu L, Zhang RY, Zhang Q, Wu ZG, Shen WF. Association of blood pressure and coronary collateralization in type 2 diabetic and nondiabetic patients with stable angina and chronic total occlusion. J Hypertens.2015; 33: 621-6.

8. Ebrahimi H, Emamian MH, Khosravi A, Hashemi H, Fotouhi A. Comparison of the accuracy of three diagnostic criteria and estimating the prevalence of metabolic syndrome: A latent class analysis. J Res Med Sci. 2019; 24: 108.

9. McEntegart MB, Badar AA, Ahmad FA, Shaukat A, MacPherson M, Irving J, Strange J, Bagnall AJ, Hanratty CG, Walsh SJ, et al. The collateral circulation of coronary chronic total occlusions. Eurolntervention. 2016; 11: e1596-603.

10. Williams B, Mancia G, Spiering W, Agabiti Rosei E, Azizi M, Burnier M, Clement DL, Coca A, deSimone G, Dominiczak A, et al. 2018 ESC/ESH Guidelines for the management of arterial hypertension. Eur Heart J. 2018; 39: 3021-3104.

11. Garber AJ, Handelsman Y, Grunberger G, Einhorn D, Abrahamson MJ, Barzilay JI, Blonde L, Bush MA, DeFronzo RA, Garber JR, et al. Consensus statement by the American association of clinical endocrinologists and American college of endocrinology on the comprehensive type 2 diabetes management algorithm-2020 executive summary. Endocr Pract. 2020;26:107-139.

12. Vo MN, Brilakis ES, Kass M, Ravandi A. Physiologic significance of coronary collaterals in chronic total occlusions. Can J Physiol Pharmacol. 2015; 93:867-71.

13. Martins CC, Bagatini MD, Simões JLB, Cardoso AM, Baldissarelli J, Dalenogare DP, Dos Santos DL, Schetinger MRC, Morsch VM. Increased oxidative stress and inflammatory markers contrasting with the activation of the cholinergic anti-inflammatory pathway in patients with metabolic syndrome. Clin Biochem. 2021; 89: 63-69.

14. Turhan H, Yasar AS, Erbay AR, Yetkin E, Sasmaz H, Sabah I. Impaired coronary collateral vessel development in patients with metabolic syndrome. Coron Artery Dis. 2005; 16: 281-5.

15. Pung YF, Chilian WM. Corruption of coronary collateral growth in metabolic syndrome: Role of oxidative stress. World J Cardiol. $2010 ; 2$ : $421-7$.

16. Joseph G, Soler A, Hutcheson R, Hunter I, Bradford C, Hutcheson B, Gotlinger KH, Jiang H, Falck JR, Proctor S, et al. Elevated 20-HETE impairs coronary collateral growth in metabolic syndrome via endothelial dysfunction. Am J Physiol Heart Circ Physiol. 2017; 312: H528-H540.

17. Piuri G, Zocchi M, Della Porta M, Ficara V, Manoni M, Zuccotti GV, Pinotti L, Maier JA, Cazzola R. Magnesium in Obesity, Metabolic Syndrome, and Type 2 Diabetes. Nutrients. 2021; 13: 320.

18. Kotsis V, Jordan J, Micic D, Finer N, Leitner DR, Toplak H, Tokgozoglu L, Athyros V, Elisaf M, Filippatos TD, et al.Obesity and cardiovascular risk: a call for action from the European Society of Hypertension Working Group of Obesity, Diabetes and the High-risk Patient and European Association for the Study of Obesity: part A: mechanisms of obesity induced hypertension, diabetes and dyslipidemia and practice guidelines for treatment. J Hypertens. 2018; 36 : 1427-1440.

19. Sasmaz H, Yilmaz MB. Coronary collaterals in obese patients: impact of metabolic syndrome. Angiology. 2009; 60: 164-8.

20. Shu W, jing J, Fu LC, Min JT, Bo YX, Ying Z, Dai CY. The relationship between diastolic pressure and coronary collateral circulation in patients with stable angina pectoris and chronic total occlusion. Am J Hypertens, 2013; 26: 630-5.

21. Börekçi A, Gür M, Şeker T, Baykan AO, Özaltun B, Karakoyun S, Karakurt A, Türkoğlu C, Makça I, Çaylı Coronary collateral circulation in patients with chronic coronary total occlusion; its relationship with cardiac risk markers and SYNTAX score. Perfusion. 2015; 30: 457-64.

22. Ito WD, Lund N, Sager H, Becker W, Wenzel U. Differential impact of diabetes mellitus type II and arterial hypertension on collateral artery growth and concomitant macrophage accumulation. Vasa. 2015; 44: 31-41.

23. Kadi H, Ozyurt H, Ceyhan K, Koc F, Celik A, Burucu T. The relationship between high-density lipoprotein cholesterol and coronary collateral circulation in patients with coronary artery disease. J Investig Med. 2012; 60: 808-12.

24. Shen Y, Chen S, Dai Y, Wang XQ, Zhang RY, Yang ZK, Hu J, Lu L, Ding FH, Shen WF. Lipoprotein (a) interactions with cholesterol-containing lipids on angiographic coronary collateralization in type 2 diabetic patients with chronic total occlusion. Cardiovasc Diabetol. $2019 ; 18: 82$.

25. Hutcheson R, Chaplin J, Hutcheson B, Borthwick F, Proctor S, Gebb S, Jadhav R, Smith E, Russell JC, Rocic P. miR-21 normalizes vascular smooth muscle proliferation and improves coronary collateral growth in metabolic syndrome. FASEB J. 2014; 28: 4088-99. 
26. Hattan N, Chilian WM, Park F, Rocic P. Restoration of coronary collateral growth in the Zucker obese rat: impact of VEGF and ecSOD. Basic Res Cardiol. 2007; 102: 217-23.

27. Dodd T, Wiggins L, Hutcheson R, Smith E, Musiyenko A, Hysell B, Russell JC, Rocic P. Impaired coronary collateral growth in the metabolic syndrome is in part mediated by matrix metalloproteinase 12-dependent production of endostatin and angiostatin. Arterioscler Thromb Vasc Biol. 2013 ; 33 : $1339-49$.

28. Yilmaz MB, Caldir V, Guray Y, Guray U, Altay H, Demirkan B, Cay S, Kisacik HL, Korkmaz S. Relation of coronary collateral vessel development in patients with a totally occluded right coronary artery to the metabolic syndrome. Am J Cardiol. 2006; 97: 636-9.

29. Mouquet F, Cuilleret F, Susen S, Sautière K, Marboeuf P, Ennezat PV, McFadden E, Pigny P, Richard F, Hennache B, et al. Metabolic syndrome and collateral vessel formation in patients with documented occluded coronary arteries: association with hyperglycaemia, insulin-resistance, adiponectin and plasminogen activator inhibitor-1. Eur Heart J. 2009; 30: 840-9.

\section{Figures}

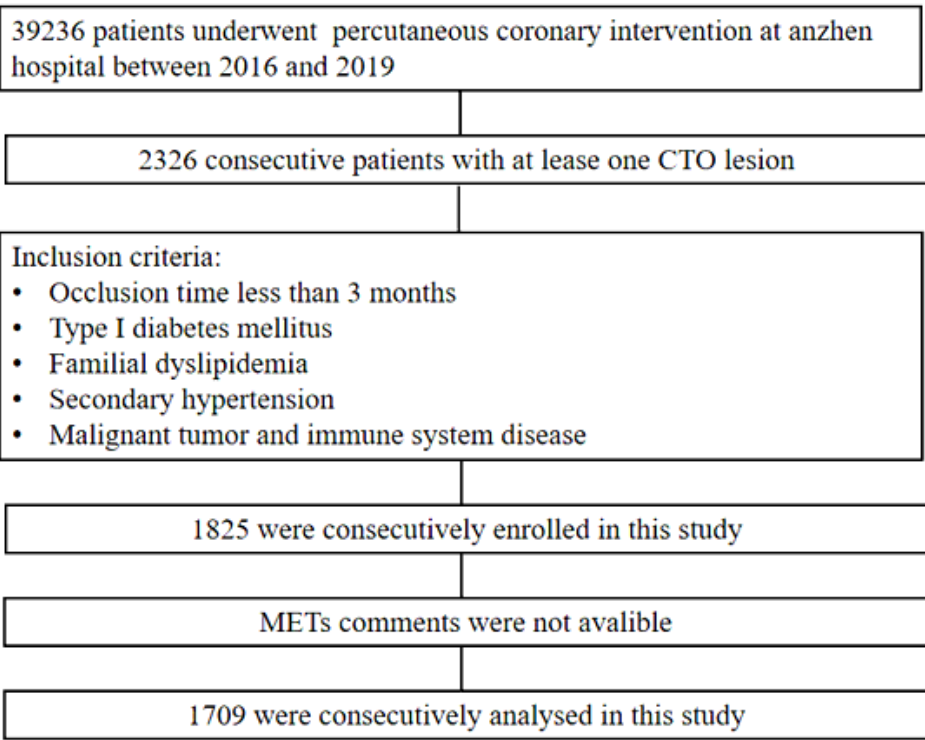

\section{Figure 1}

Population flowchart of enroll patients.

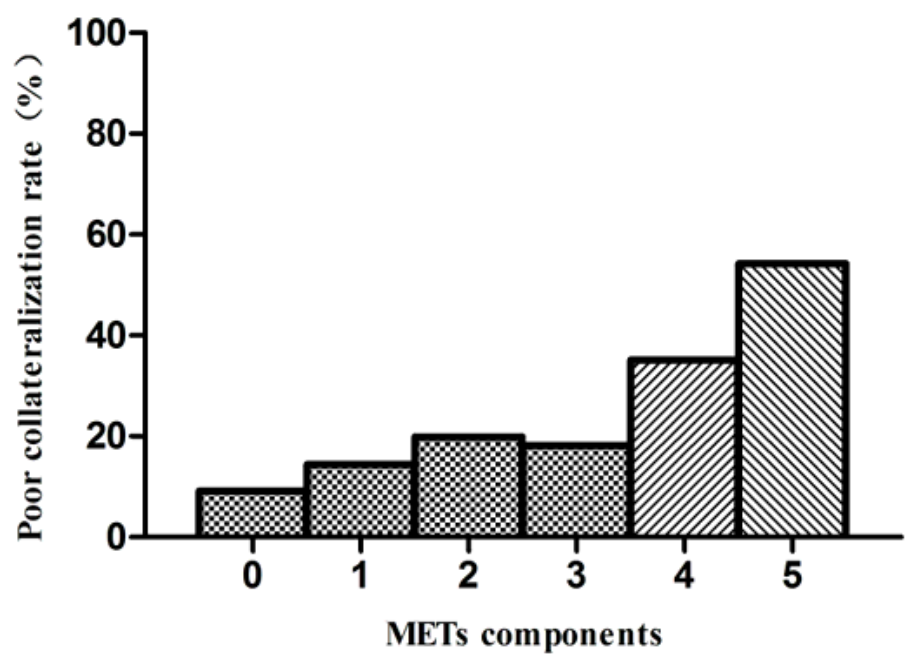

Figure 2

Poor collateralization of metabolic syndrome components. 


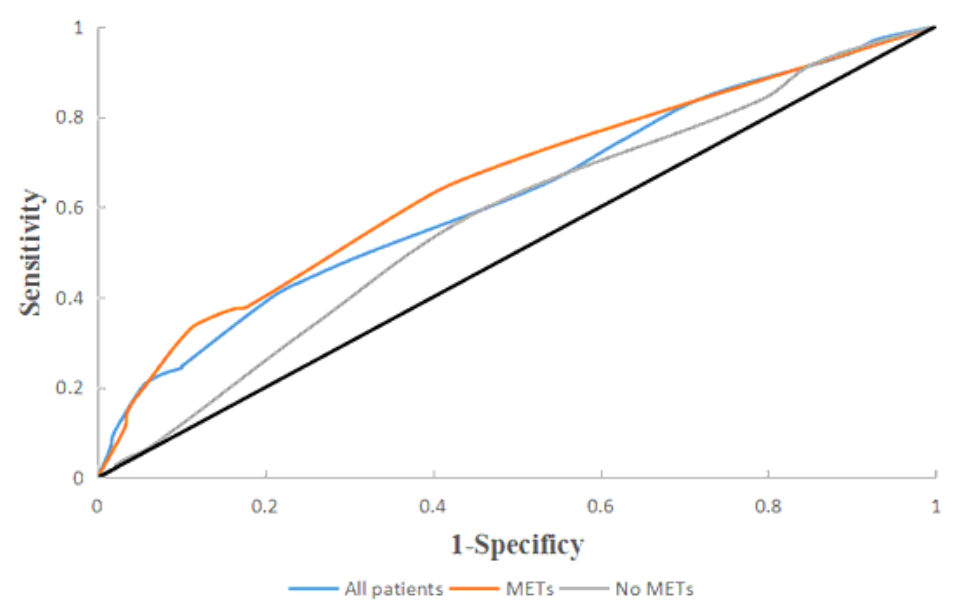

\section{Figure 3}

ROC curve analysis of the score system for the prediction of poor collateralization. 